\title{
Synthesis, Crystal Structure, Vibrational Study and DFT Computation of Barium Dihydrogenomonophosphate $\mathrm{Ba}\left(\mathrm{H}_{2} \mathrm{PO}_{4}\right)$
}

\author{
Rachida Oubouaza1, Mark Benson ${ }^{2}$, Jakub Wojciechowski $^{3}$, Samir Chtita ${ }^{4}$, Malika Tridane $^{5}$, Said Belaaouad $^{6}$ \\ ${ }^{1}$ Laboratory of chemistry-physics of materials LCPM, Faculty of Sciences Ben M'Sik, B.P7955, Hassan II University of Casablanca, Morocco; \\ ${ }^{2}$ Institute of General and Ecological Chemistry, Lód'z University of Technology, 116 Zeromskiego St., 90-924 Lodz, Poland; \\ ${ }^{3}$ Department of Chemistry, University of Liverpool, CrownStreet, Liverpool L697ZD, UK; \\ ${ }^{4}$ Laboratory of chemistry-physics of materials LCPM, Faculty of Sciences Ben M'Sik, B.P7955, Hassan II University of Casablanca, Morocco; \\ ${ }^{5}$ Regional Center of the education and training trades Anfa Bd BirAnzarane Casablanca. Morocco; \\ ${ }^{6}$ Laboratory of chemistry-physics of materials LCPM, Faculty of Sciences Ben M'Sik, B.P7955, Hassan II University of Casablanca, Morocco;
}

oubouazarachida01@gmail.com

The single crystal of barium dihydrogenomonophosphate, $\mathrm{Ba}\left(\mathrm{H}_{2} \mathrm{PO}_{4}\right)_{2}$ was prepared by the direct method. This compound exists in two forms: one orthorhombic, the other triclinic. In this work, we are interested in the triclinic form from the vibrational and crystalline sides too.X-ray crystallography showed that this compound crystallizes in the triclinic centrosymmetric with space group P-1 $(Z=2)$ with $\mathrm{a}=6.9917(5) \AA, \mathrm{b}=7.1929(5) \AA, \mathrm{c}=7.9667(9) \AA, \alpha=104.517(8)^{\circ}, \beta=95.918(7)^{\circ}$ and $\gamma=109.459(6)$. The structure was solved from 3444 independent reflections with $\mathrm{R}=0.0198$ with $\mathrm{wR}=0.0633$. The bands observed in the infrared and Raman spectra of $\mathrm{Ba}\left(\mathrm{H}_{2} \mathrm{PO}_{4}\right)_{2}$ are assigned based on the literature results and the theorical group analyses carried out in the group of factors $\mathrm{Ci}$. We were based on density functional theory (DFT / B3LYP) methods with the LanL2DZ base set for the calculation of optimal molecular geometry, harmonic vibration frequencies, infrared intensities and Raman scattering activities. The HOMO-LUMO properties and geometries of this compound have been determined and discussed. The computational structural parameters are generally in agreement with the experimental investigations. The theoretical infrared and Raman spectra for the title compound have been constructed.

Keywords: crystal structures; X-ray diffraction; Raman; Infrared; DFT; barium dihydrogenomonophosphate. 